\title{
Autism, Epilepsy and Intellectual Disability: A Clinical Conundrum
}

\author{
Vaishakh Anand ${ }^{1} \cdot$ Prashant Jauhari $^{1}$ (B)
}

Received: 18 July 2019 / Accepted: 18 July 2019/Published online: 31 July 2019

(C) Dr. K C Chaudhuri Foundation 2019

Autism and epilepsy are heterogenous disorders of neuronal networking. Both these disorders along with global developmental delay/intellectual disability (GDD/ID) often co-exist in children. These kids have a worse neurocognitive outcome than those with autism or epilepsy alone. Recent concepts of epileptic encephalopathy, shared molecular mechanisms of interneuronal (GABAergic) dysfunction and common genetic etiologies have unified these previously distinct clinical entities. The world literature reports that epilepsy may be prevalent in up to a third of children with autism [1]. The exact figure however depends upon the diagnostic criteria used for autism spectrum disorder, EEG protocol, age group of cases and prevalence of other co-morbid conditions such as GDD/ID.

Data originating from the Indian subcontinent is scanty. Anukirthiga and colleagues have tried to fill this lacuna. They studied the prevalence of epilepsy and interictal epileptiform discharges in children with autism and Attention deficit hyperactivity disorder (ADHD). The important observations of their study were: (A) A high prevalence of epilepsy in autism (45.5\%) and ADHD (37.5\%), (B) A strong association of epilepsy in autism with low IQ as well as severity of autism and, (C) A significant proportion of cases who did not have seizures had interictal epileptiform EEG discharges 17/90 (19\%) [2].

The clinical connotation of Anukirthiga et al. study is perplexing. It is unclear whether autism leads to epilepsy or it is the other way around. Similarly, whether the combination of the two leads to cognitive decline or a significantly low intellect is an independent risk factor for epilepsy in autism, is debatable [3]. Moreover, a pandora of unanswered clinically relevant questions such as what risk factors promote epilepsy in autism; whether to screen all autistic children with an EEG;

Prashant Jauhari

pjauhari0@gmail.com

1 Child Neurology Division, Department of Pediatrics, All India Institute of Medical Sciences, New Delhi 110029, India what to do if an abnormality is detected on EEG, especially when clinical seizures are absent, make a protocolized management plan elusive. Having said that, an old concept of essential and complex autism may help in clinical decision making. Pediatricians should consider autism as an age dependent symptom complex of multifactorial and diverse etiology which either manifests alone as 'essential' autism or as a part of a more pervasive disorder such as tuberous sclerosis, electrical status epilepticus in sleep (ESES), progressive neurodegenerative or metabolic disorders [4]. In the latter type of presentation, there should be a high index of suspicion for co-morbid conditions including epilepsy and a low threshold for treatment of the same [3]. For those presenting with 'essential' autism, EEG should be reserved in cases with seizures or events that may represent seizures or with clear language regression [5].

Another problem with screening EEG as evident in Anukirthiga et al. study is that EEG abnormalities do not always corelate with seizures [2]. Along with it, diagnosing seizure in autism may be difficult as the behavioral and motor stereotypes associated with some seizure types may mimic autistic behavior traits. Due to this ambiguity, guidelines discourage screening EEG in absence of clinical seizure [3]. If a clinician decides to order an EEG, it should preferably be a $30 \mathrm{~min}$ sleep record with arousal/awake response with or without intermittent photic stimulation. This protocol will be able to identify EEG patterns which are highly suggestive of an epileptic encephalopathy irrespective of clinical seizures. One such pattern is that of ESES which is characterized by sleep potentiated frontal or temporal dominant spike wave discharges with spike wave index $>50$ [6]. Photoparoxysmal response in EEG in autism may suggest a diagnosis of Angelman syndrome, late infantile neuronal ceroid lipofuscinosis or $\mathrm{SCN} 1 \mathrm{~A}$ genetic variation. Identifying these conditions is important as the treatment plan completely differs. Early consideration of antiepileptic drugs, immunomodulation and dietary therapy may be more beneficial.

The study infers that an active surveillance for epilepsy in children with autism is required. Decision to perform EEG at 
present should be taken on case-to-case basis and epileptiform abnormalities should be interpreted cautiously.

\section{References}

1. Lai M-C, Lombardo MV, Baron-Cohen S. Autism. Lancet. 2014;383:896-910.

2. Anukirthiga B, Mishra D, Pandey S, Juneja M, Sharma N. Prevalence of epilepsy and inter-ictal epileptiform discharges in children with autism and attention-deficit hyperactivity disorder. Indian J Pediatr. 2019. https://doi.org/10.1007/s12098-019-02977-6.

3. Thodeson DM, Dowd D, Golla G, Evans P, Huang R, Sirsi D. Evolution of EEG findings in children with autism spectrum disorder: a tertiary care centre's clinical experience. J Psychol Psychiatry. 2018;2:1-4.

4. Miles JH, Takahashi TN, Bagby S, et al. Essential versus complex autism: definition of fundamental prognostic subtypes. Am J Med Genet A. 2005;135:171-80.

5. Johnson CP. Myers SM, and the council on children with disabilities. Identification and evaluation of children with autism spectrum disorders. Pediatrics. 2007;120:1183-215.

6. Scheltens-de BM. Guidelines for EEG in encephalopathy related to ESES/CSWS in children. Epilepsia. 2009;50:13-7.

Publisher's Note Springer Nature remains neutral with regard to jurisdictional claims in published maps and institutional affiliations. 